\title{
METHOD OF COOPERATIVE FORMATION CONTROL FOR UNDERACTUATED USVS BASED ON NONLINEAR BACKSTEPPING AND CASCADE SYSTEM THEORY
}

\author{
Zaopeng Dong ${ }^{1,2}$ \\ Yang Liu ${ }^{1,2}$ \\ Hao Wang ${ }^{1,2}$ \\ Tao Qin ${ }^{3 *}$ \\ ${ }^{1}$ Key Laboratory of High Performance Ship Technology (Wuhan University of Technology), Ministry of Education, \\ Wuhan University of Technology, Wuhan, China \\ ${ }^{2}$ School of Transportation, Wuhan University of Technology, Wuhan, China \\ ${ }^{3}$ School of Mechanical Engineering, Hubei University of Arts and Sciences, Xiangyang, China
}

*Corresponding author: e-mail: heu_qt@163.com (T. Qin)

\begin{abstract}
This paper presents a method for the cooperative formation control of a group of underactuated USVs. The problem of formation control is first converted to one of stabilisation control of the tracking errors of the follower USVs using system state transformation design. The followers must keep a fixed distance from the leader USV and a specific heading angle in order to maintain a certain type of formation. A global differential homeomorphism transformation is then designed to create a tracking error system for the follower USVs, in order to simplify the description of the control system. This makes the complex formation control system easy to analyse, and allows it to be decomposed into a cascaded system. In addition, several intermediate state variables and virtual control laws are designed based on nonlinear backstepping, and actual control algorithms for the follower USV to control the surge force and yaw moment are presented. A global system that can ensure uniform asymptotic stability of the USV' cooperative formation control is achieved by combining Lyapunov stability theory and cascade system theory. Finally, several simulation experiments are carried out to verify the validity, stability and reliability of our cooperative formation control method.
\end{abstract}

Keywords: unmanned surface vehicle (USV),cooperative formation control,underactuated system,nonlinear backstepping,cascade system theory

\section{INTRODUCTION}

An unmanned surface vehicle (USV) is an intelligent autonomous surface vessel, of a type that has played an indispensable role in several fields such as science, economics and the military [1-3]. The problem of cooperative formation tracking control of multiple USVs has attracted increasing amounts attention from researchers from all over the world over recent years, since a team of USVs working together is often more effective than a single vehicle for challenging missions such as surveillance, hydrographic surveys, autonomous exploration of ocean resources, reconnaissance, rescue operations and perimeter security [4-6]. It is well-known that the control system of an USV is generally underactuated, since the number of control inputs is less than the degrees of freedom and there is an unintegrable acceleration constraint on the system. Kinematic and dynamic models of cooperative formation tracking for USVs are highly nonlinear and coupled [7-10]; this means that classic linear methodologies cannot be applied, and more advanced methods need to be developed to achieve cooperative formation tracking control of USVs.

Several exploratory algorithms have been proposed for this problem. A leader-follower formation tracking control algorithm was presented for an underactuated USV in [2, 11], in which a radial basis function neural network and adaptive robust control techniques were adopted to preserve the robustness of the controller against unmodelled dynamics and environmental disturbances induced by waves and ocean 
currents. Another control strategy was proposed in [12], in which each follower was designed to track a virtual vehicle and the formation control problem was transformed into a set of position (including heading) tracking problems. In [13], cooperative formation control was formulated as an adaptive feedback control problem for a line of sight (LOS)-based configuration of a leader and a follower, and asymmetric barrier Lyapunov functions were used in the design of the controller to account for time-varying constraints on the LOS and bearing angle. An approach based on model prediction control was proposed in [14] to address the vessel train formation problem. This method considered cooperative collision avoidance and the grouping of vessels, and a singlelayer serial iterative architecture was adopted to reduce the communication requirements and ensure robustness against failure. The leader-follower formation control problem for USVs with unknown nonlinear dynamics and actuator faults was addressed in [15], and a low-complexity prescribed performance controller was proposed without the help of auxiliary neural/fuzzy systems or adaptive mechanisms. The authors of [16] explored the problem of finite-time extended state observer-based distributed formation control for USVs with input saturation and external disturbances, and a novel finite-time extended state observer was proposed to estimate the unavailable velocity measurements and external disturbances. A novel nonlinear sliding mode control method for dealing with the formation control of underactuated ships was presented in [17], in which the state space of the system was partitioned into two regions, and in [18], the dynamic equations for the position and attitude were analysed using a coordinate transformation with the aid of the backstepping technique. In [19], the platoon formation control problem for USVs in the presence of modelling uncertainties and time-varying external disturbances was studied, and performance guarantees were enforced in the control design to provide transient performance specifications for formation errors, including errors related to the LOS range and angle. A novel robust adaptive formation control scheme based on a minimal learning parameter (MLP) algorithm and a disturbance observer (DOB) was presented in [20], and a novel disturbance rejection control was designed in [21] that took into account the disturbance caused by the formation adjustment among ships. A system for motion planning, collision avoidance, guidance and control for a formation of autonomous surface vehicles navigating in a complex marine environment was presented in [6]; the motion planning unit was based on an angle-guidance fastmarching square method, while the control unit is composed of a PID heading controller and a speed controller. The authors of [4] presented a coordinated tracking strategy with swarm centre identification, self-organised aggregation, collision avoidance and a distributed controller design for multiple USVs, while an adaptive observer based on a neural network was designed to estimate the velocity information of USVs in [22]. Decentralised finite time formation control of underactuated USVs in the presence of model uncertainty and environmental disturbance was addressed in [23], and in [24], the authors presented an approach for the distributed timevarying formation control of a swarm of underactuated USVs subject to unknown input gains, model uncertainties and ocean disturbances. A robust control scheme was developed for the time-varying formation of multiple underactuated autonomous underwater vehicles (AUVs) with environment disturbances and input saturation in [25]. A new robust model predictive control (MPC) algorithm for trajectory tracking of an autonomous surface vehicle (ASV) in the presence of time-varying external disturbances was proposed in [5], and a high-performance super-twisting sliding mode control method for a maritime autonomous surface ship (MASS) using approximate dynamic programming (ADP)-based adaptive gains and time delay estimation was presented in [26]. Although MPC is a superior method for motion control of a ship, especially when the model is unknown, the design process of constraint conditions is strict and the calculation of the system is complex in some cases.

Despite the multitude of research results in the literature on the cooperative formation control of USVs, the design process of the controller is often too complicated for calculation and analysis, and the performance of the controller needs to be further improved. In this paper, we present innovative work on the transformation of the cooperative formation control problem, and propose some design ideas for maintaining a formation and applying control methods and stability theories. Compared with existing studies, the main contributions of this paper can be summarised as follows: (i) we present a novel description of the cooperative formation control problem for a group of USVs, in which the desired positions and attitude angles of the follower USVs are transformed into intermediate variables that can help in the design of the controller; (ii) we design a new kind of global differential homeomorphism transformation for the tracking error system of the follower USVs, which simplifies the description of the control system, thus making the complex formation control system easy to analyse and allowing it to be decomposed into a cascaded system; (iii) we propose an improved controller for the cooperative formation control of a group of underactuated USVs by combining a backstepping technique with Lyapunov's direct method and cascade system theory, and devise some intermediate state variables and virtual control laws for the design process of the control algorithm.

\section{PROBLEM FORMULATION}

\section{A. USV MODELING}

When designing a cooperative formation controller for a group of underactuated USVs, mathematical models are used, including kinematic and dynamic models, which can be expressed as follows [27]: 


$$
\left\{\begin{array}{l}
\dot{x}_{j}=u_{j} \cos \varphi_{j}-v_{j} \sin \varphi_{j} \\
\dot{y}_{j}=u_{j} \sin \varphi_{j}+v_{j} \cos \varphi_{j} \\
\dot{\varphi}_{j}=r_{j} \\
\dot{u}_{j}=\frac{m_{22 j}}{m_{11 j}} v_{j} r_{j}-\frac{d_{11 j}}{m_{11 j}} u_{j}+\frac{1}{m_{11 j}} \tau_{u j} \\
\dot{v}_{j}=-\frac{m_{11 j}}{m_{22 j}} u_{j} r_{j}-\frac{d_{22 j}}{m_{22 j}} v_{j} \\
\dot{r}_{j}=\frac{m_{11 j}-m_{22 j}}{m_{33 j}} u_{j} v_{j}-\frac{d_{33 j}}{m_{33 j}} r_{j}+\frac{1}{m_{33 j}} \tau_{r j}
\end{array}\right.
$$

where $j=k+1, k=1, \ldots, N$. This means that there is one leader USV and $N$ follower USVs in this group. $x_{j}$ and $y_{j}$ denote the position coordinates of each USV in the earth-fixed frame; $\varphi_{j}$ is the heading angle; $u_{j}, v_{j}$ and $r_{j}$ represent the velocity vectors for each USV in the surge, sway and yaw directions in the body-fixed frame, respectively. The surge force $\tau_{u j}$ and the yaw moment $\tau_{r j}$ are considered as control inputs which drive the USV to move. $m_{11 j}, m_{22 j}, m_{33 j}$ express the inertia coefficients of the USV, including added mass effects, and $d_{11 j}, d_{22 j}, d_{33 j}$ represent hydrodynamic damping coefficients.

\section{B. PROBLEM FORMULATION}

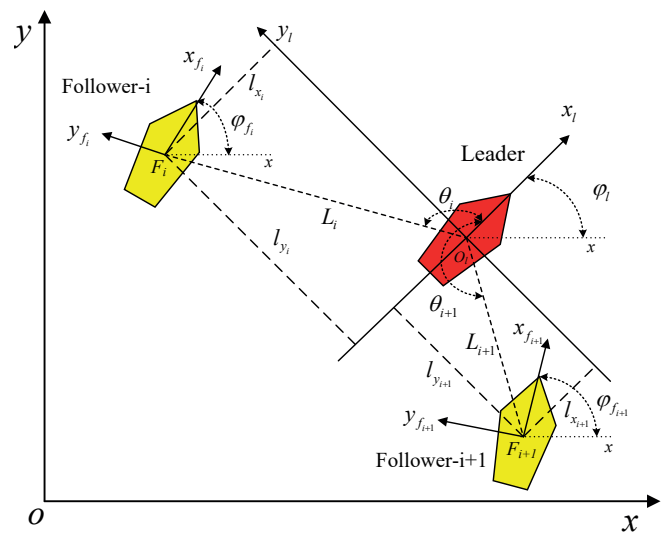

Fig. 1 Cooperative formation control of USVs based on a leader-follower scheme

The cooperative formation control of a group of USVs using a leader-follower approach is illustrated in Fig. 1, which shows one leader and two followers. As we can see from the figure, $\left\{x_{l} o_{l} y_{l}\right\}$ denotes the body-fixed frame of the leader USV, while $\left\{x_{f_{i}} F_{i} y_{f_{i}}\right\}$ and $\left\{x_{f_{t+1}} F_{i+1} y_{f_{t+1}}\right\}$ represent the body-fixed frames of the two follower USVs, and $\varphi_{l}, \varphi_{f_{i}}, \varphi_{f_{i+1}}$ indicate their heading angles. All of the headings are defined in the same direction, with a rotation from the earth-fixed frame to the body-fixed frame; the clockwise direction is negative, and the counterclockwise direction is positive. The leaderfollower scheme for cooperative formation control of the USVs can then be formulated as follows: if the distance between each follower $i$ and the leader $L_{i}$, and the angle $\theta_{i}$ between the lines $o_{l} x_{l}$ and $o_{l} F_{i}$ shown in Fig. 1, can be kept to certain values, then the follower and the leader will sail in a specific formation, and cooperative formation control is achieved.

We assume that the desired trajectory, heading angle and velocities of the leader USV can be expressed as $\left\{x_{l d}, y_{l d}, \varphi_{l d}, u_{l d}, v_{l d}, r_{l d}\right\}$, while the desired state of the follower USV can be represented as $\left\{x_{f_{i} d}, y_{f_{i} d}, \varphi_{f_{i} d}, u_{f_{i} d}, v_{f_{i} d}, r_{f_{i} d}\right\}$. From the geometric description in Fig. 1, we can deduce the following mathematical expression, in which the heading angles are defined in the range from $-\pi$ to $\pi$, and the positions of the leader USV and the follower USVs can be arbitrarily chosen according to the requirements of the task:

$$
\left\{\begin{array}{l}
l_{x_{i}}=\left(x_{f_{i}}-x_{l}\right) \cos \varphi_{l}+\left(y_{f_{i}}-y_{l}\right) \sin \varphi_{l} \\
l_{y_{i}}=-\left(x_{f_{i}}-x_{l}\right) \sin \varphi_{l}+\left(y_{f_{i}}-y_{l}\right) \cos \varphi_{l} \\
\varphi_{i}=\varphi_{f_{i}}-\varphi_{l}
\end{array}\right.
$$

We can see that $L_{i}$ and the angle $\theta_{i}$ should be kept at certain values, and this also means that $l_{x_{i}}, l_{y_{i}}, \varphi_{i}$ need to be maintained in a specific state. The desired outcome can be expressed as $l_{x_{d} d}, l_{y_{d}}, \varphi_{i d}$. We then obtain the following equations, which express the desired stable positional relationship between the leader and follower USVs when USVs' formation control has been realised:

$$
\left\{\begin{array}{l}
l_{x_{i} d}=\left(x_{f_{i} d}-x_{l d}\right) \cos \varphi_{l d}+\left(y_{f_{i} d}-y_{l d}\right) \sin \varphi_{l d} \\
l_{y_{i} d}=-\left(x_{f_{i} d}-x_{l d}\right) \sin \varphi_{l d}+\left(y_{f_{i} d}-y_{l d}\right) \cos \varphi_{l d} \\
\varphi_{i d}=\varphi_{f_{i} d}-\varphi_{l d}
\end{array}\right.
$$

The following expressions can be obtained from a system state transformation:

$$
\left\{\begin{array}{l}
x_{f_{i} d}=l_{x_{i} d} \cos \varphi_{l d}-l_{y_{i} d} \sin \varphi_{l d}+x_{l d} \\
y_{f_{i} d}=l_{x_{i} d} \sin \varphi_{l d}+l_{y_{i} d} \cos \varphi_{l d}+y_{l d} \\
\varphi_{f_{i} d}=\varphi_{i d}+\varphi_{l d}
\end{array}\right.
$$

In order to keep the overall formation of the USVs in line with the expected form, the real state variables for the leader USV, such as its position, heading angle and velocities $\left\{x_{l}, y_{l}, \varphi_{l}, u_{l}, v_{l}, r_{l}\right\}$, are used as a reference for the desired values $\left\{x_{l d}, y_{l d}, \varphi_{l d}, u_{l d}, v_{l d}, r_{l d}\right\}$. To achieve overall cooperative formation control, the desired position and heading angle of follower $i$ can be described as follows, i.e. the desired state of the follower USVs that will be used in the actual formation tracking control process:

$$
\left\{\begin{array}{l}
x_{f_{i} d}=l_{x_{i} d} \cos \varphi_{l}-l_{y_{i} d} \sin \varphi_{l}+x_{l} \\
y_{f_{i} d}=l_{x_{i} d} \sin \varphi_{l}+l_{y_{i} d} \cos \varphi_{l}+y_{l} \\
\varphi_{f_{i} d}=\varphi_{i d}+\varphi_{l}
\end{array}\right.
$$


Taking derivatives of both sides of the above equations and applying a mathematical conversion gives:

$$
\left\{\begin{array}{l}
\dot{x}_{f_{i} d}=-r_{l} l_{x_{i} d} \sin \varphi_{l}-r_{l} l_{y_{i} d} \cos \varphi_{l}+u_{l} \cos \varphi_{l}-v_{l} \sin \varphi_{l} \\
\dot{y}_{f_{i} d}=r_{l} l_{x_{i} d} \cos \varphi_{l}-r_{l} l_{y_{i} d} \sin \varphi_{l}+u_{l} \sin \varphi_{l}+v_{l} \cos \varphi_{l} \\
\dot{\varphi}_{f_{i} d}=r_{l}
\end{array}\right.
$$

The desired velocities of a follower $i$ can be expressed as:

$$
\left\{\begin{array}{l}
u_{f_{i} d}=\dot{x}_{f_{i} d} \cos \varphi_{f_{i} d}+\dot{y}_{f_{i} d} \sin \varphi_{f_{i} d}=r_{l} l_{x_{i} d} \sin \varphi_{i d}-r_{l} l_{y_{i} d} \cos \varphi_{i d}+u_{l} \cos \varphi_{i d}+v_{l} \sin \varphi_{i d} \\
v_{f_{i} d}=-\dot{x}_{f_{d} d} \sin \varphi_{f_{i} d}+\dot{y}_{f_{i} d} \cos \varphi_{f_{i} d}=r_{l} l_{x_{i} d} \cos \varphi_{i d}+r_{l} l_{y_{i} d} \sin \varphi_{i d}-u_{l} \sin \varphi_{i d}+v_{l} \cos \varphi_{i d} \\
r_{f_{i} d}=\dot{\varphi}_{f_{i} d}
\end{array}\right.
$$

In this way, the problem of formation control of the underactuated USVs can be converted to the problem of designing control inputs $\left(\tau_{u i}, \tau_{r i}\right)$ for each individual follower USV. This can help in achieving the following goal:

$$
\lim _{t \rightarrow \infty}\left\|\eta_{i}-\eta_{i d}\right\|=0
$$

where $\eta_{i}=\left[x_{f_{i}}, y_{f_{i}}, \varphi_{f_{i}}, u_{f_{i}}, v_{f_{i}}, r_{f_{i}}\right]$ are the real state variables of the follower USV $i$, including its position, heading angle and velocities, while $\eta_{i d}=\left[x_{f_{d}}, y_{f_{f}}, \varphi_{f_{d}}, u_{f_{d}}, v_{f_{f}}, r_{f_{f d}}\right]$ represent the desired state variables of the USV, as shown below:

$$
\left\{\begin{array}{l}
x_{f_{i} d}=l_{x_{i} d} \cos \varphi_{l}-l_{y_{i} d} \sin \varphi_{l}+x_{l} \\
y_{f_{i} d}=l_{x_{i} d} \sin \varphi_{l}+l_{y_{i} d} \cos \varphi_{l}+y_{l} \\
\varphi_{f_{i} d}=\varphi_{i d}+\varphi_{l} \\
u_{f_{i} d}=r_{l} l_{x_{i} d} \sin \varphi_{i d}-r_{l} l_{y_{i} d} \cos \varphi_{i d}+u_{l} \cos \varphi_{i d}+v_{l} \sin \varphi_{i d} \\
v_{f_{i} d}=r_{l} l_{x_{i} d} \cos \varphi_{i d}+r_{l} l_{y_{i} d} \sin \varphi_{i d}-u_{l} \sin \varphi_{i d}+v_{l} \cos \varphi_{i d} \\
r_{f_{i} d}=\dot{\varphi}_{f_{i} d}
\end{array}\right.
$$

\section{CONTROLLER DESIGN}

In this section, a cooperative formation control algorithm is proposed for underactuated USVs based on nonlinear backstepping and cascade system theory. The main process of this algorithm is illustrated in Fig. 2.
The expected tracking state information for the follower USV can be obtained based on the state information for the leader USV and the desired formation. The intermediate variables can then be designed and the tracking errors obtained, and the model of the cooperative formation control error system can be established. Finally, the control algorithm is designed based on nonlinear backstepping and cascade system theory.

\section{A. GLOBAL DIFFERENTIAL HOMEOMORPHISM TRANSFORMATION DESIGN}

In order to create a better description and to carry out an analysis of the state variables of the follower USV, the following differential homeomorphic intermediate state variables $z_{n f}, n=1, \ldots, 6$ are proposed based on the kinematics and dynamics equations expressed in (1). The main goal is to transform the system into a form that can be expressed as a cascading system.

$$
\left\{\begin{array}{l}
z_{1 f_{i}}=x_{f_{i}} \cos \varphi_{f_{i}}+y_{f_{i}} \sin \varphi_{f_{i}} \\
z_{2 f_{i}}=-x_{f_{i}} \sin \varphi_{f_{i}}+y_{f_{i}} \cos \varphi_{f_{i}}+m_{22 f_{i}} v_{f_{i}} / d_{22 f_{i}} \\
z_{3 f_{i}}=\varphi_{f_{i}} \\
z_{4 f_{i}}=-m_{11 f_{i}} u_{f_{i}} / d_{22 f_{i}}-x_{f_{i}} \cos \varphi_{f_{i}}-y_{f_{i}} \sin \varphi_{f_{i}} \\
z_{5 f_{i}}=v_{f_{i}} \\
z_{6 f_{i}}=r_{f_{i}}
\end{array}\right.
$$

Differentiating each equation in (10) leads to the following expressions:

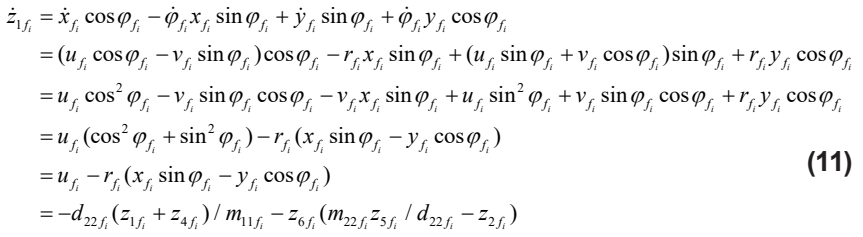

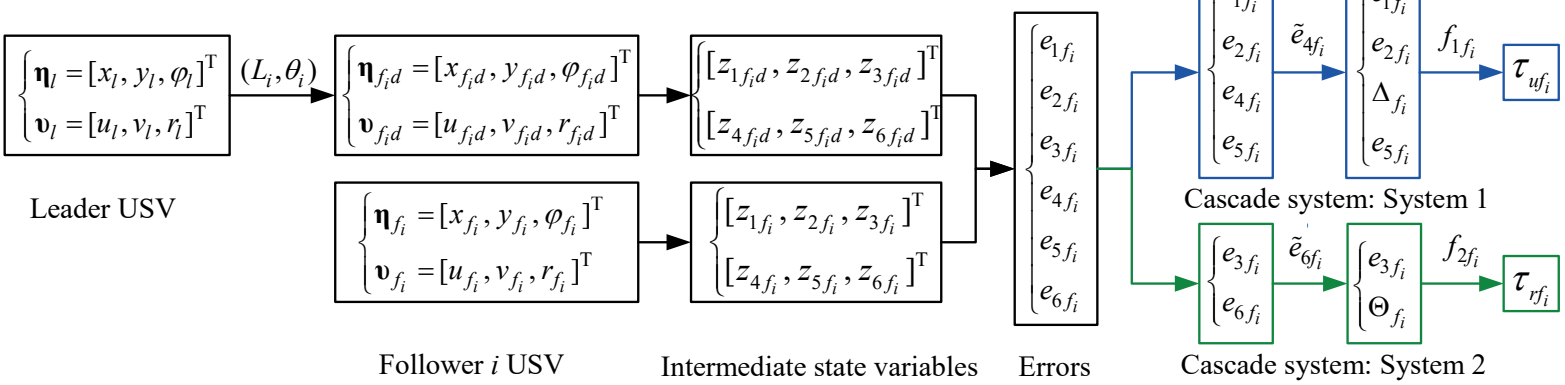

Fig. 2. Main process of the controller algorithm 


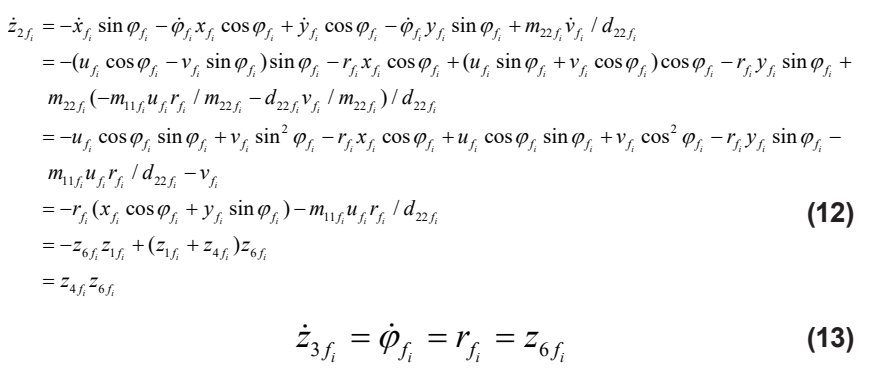

$$
\begin{aligned}
\dot{z}_{4 f_{i}} & =-m_{11 f_{i}} \dot{u}_{f_{i}} / d_{22 f_{i}}-\dot{z}_{1 f_{i}} \\
& =-m_{11 f_{i}} \dot{u}_{f_{i}} / d_{22 f_{i}}-\left(-d_{22 f_{i}} z_{1 f_{i}} / m_{11 f_{i}}-d_{22 f_{i}} z_{4 f_{i}} / m_{11 f_{i}}+z_{2 f_{i}} z_{6 f_{i}}-d_{22 f_{i}} z_{5 f_{i}} z_{6 f_{i}} / m_{22 f_{i}}\right) \\
& =-m_{11 f_{i}} \dot{u}_{f_{i}} / d_{22 f_{i}}+d_{22 f_{i}}\left(z_{1 f_{i}}+z_{4 f_{i}}\right) / m_{11 f_{i}}-z_{2 f_{i}} z_{6 f_{i}}+m_{22 f_{i}} z_{5 f_{i}} z_{6 f_{i}} / d_{22 f_{i}}
\end{aligned}
$$

$$
\begin{aligned}
\dot{z}_{5 f_{i}} & =-m_{11 f_{i}} u_{f_{i}} r_{f_{i}} / m_{22 f_{i}}-d_{22 f_{i}} v_{f_{i}} / m_{22 f_{i}} \\
& =-m_{11 f_{i}}\left[-d_{22 f_{i}}\left(z_{1 f_{i}}+z_{4 f_{i}}\right) / m_{11 f_{i}}\right] z_{6 f_{i}} / m_{22 f_{i}}-d_{22 f_{i}} z_{5 f_{i}} / m_{22 f_{i}} \\
& =d_{22 f_{i}}\left(z_{1 f_{i}}+z_{4 f_{i}}\right) z_{6 f_{i}} / m_{22 f_{i}}-d_{22 f_{i}} z_{5 f_{i}} / m_{22 f_{i}}
\end{aligned}
$$

$$
\dot{z}_{6 f_{i}}=\dot{r}_{f_{i}}
$$

Using equations (14) and (16), the virtual intermediate control input variables, $f_{1 f_{i}}$ and $f_{2 f_{i}}$, can be expressed as follows:

$\left\{f_{1 f_{i}}=-m_{11 f_{i}} \dot{u}_{f_{i}} / d_{22 f_{i}}+d_{22 f_{i}}\left(z_{1 f_{i}}+z_{4 f_{i}}\right) / m_{11 f_{i}}-z_{2 f_{i}} z_{6 f_{i}}+m_{22 f_{i}} z_{5 f_{i}} z_{6 f_{i}} / d_{22 f_{i}}\right.$ $\left\{f_{2 f_{i}}=\dot{r}_{f_{i}}\right.$

Based on the above design and analysis, our mathematical model of the USVs can be converted into the following form, which is differentially homeomorphic with the system expressed in (1):

$$
\left\{\begin{array}{l}
\dot{z}_{1 f_{i}}=-d_{22 f_{i}} z_{1 f_{i}} / m_{11 f_{i}}-d_{22 f_{i}} z_{4 f_{i}} / m_{11 f_{i}}+z_{2 f_{i}} z_{6 f_{i}}-m_{22 f_{i}} z_{5 f_{i}} z_{6 f_{i}} / d_{22 f_{i}} \\
\dot{z}_{2 f_{i}}=z_{4 f_{i}} z_{6 f_{i}} \\
\dot{z}_{3 f_{i}}=z_{6 f_{i}} \\
\dot{z}_{4 f_{i}}=f_{1 f_{i}} \\
\dot{z}_{5 f_{i}}=d_{22 f_{i}}\left(z_{1 f_{i}}+z_{4 f_{i}}\right) z_{6 f_{i}} / m_{22 f_{i}}-d_{22 f_{i}} z_{5 f_{i}} / m_{22 f_{i}} \\
\dot{z}_{6 f_{i}}=f_{2 f_{i}}
\end{array}\right.
$$

\section{B. DEVELOPING THE TRACKING ERROR SYSTEM}

The expected trajectory of the follower USV $i$ described in (9) also needs to comply with the kinematic and dynamic expressions in (1), since otherwise the required trajectory will not be followed. The desired state variables and control inputs of follower $i$ described in (9), namely $\left(x_{f_{d} d}, y_{f_{d d}}, \varphi_{f_{d}}, u_{f_{d} d}, v_{f_{d}}, r_{f_{d} d}\right)$ and $\left(\tau_{u f f d}, \tau_{r f, d}\right)$, could be similarly transformed to $z_{n f, d}, n=1, \ldots, 6$ and $\left(f_{1 f_{d}}, f_{2 f_{d} d}\right)$ as shown in expressions (10) and (17). Thus, the desired trajectory of the follower $i$ can be expressed as follows:

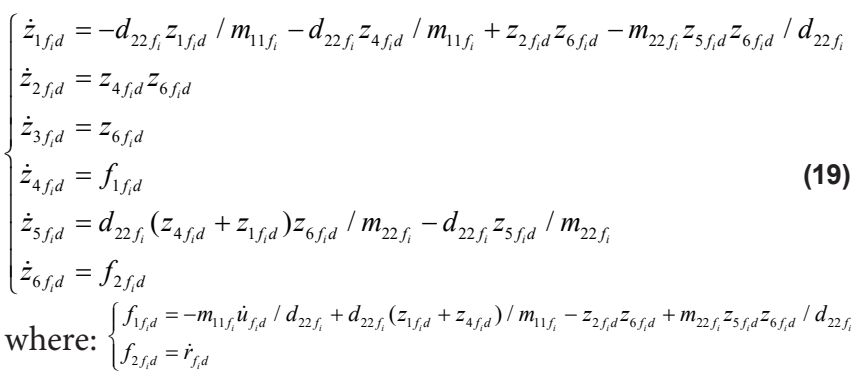

We define the following cooperative formation tracking control error variables for follower $i$ :

$$
e_{n f_{i}}=z_{n f_{i}}-z_{n f_{i} d}, n=1, \ldots, 6
$$

Differentiation and analysis of the error variables in (20) leads to the following equations for the cooperative formation tracking control error system:

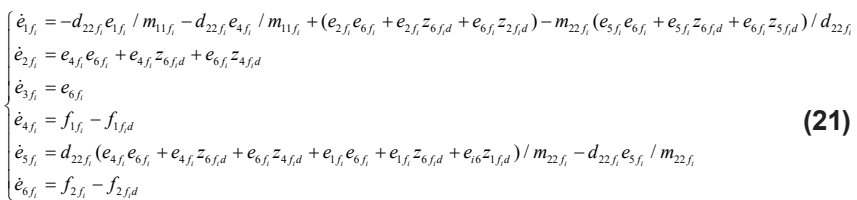

This means that if the cooperative formation-tracking control error system mentioned above can be stabilised globally and asymptotically, we can achieve stable cooperative formation control of the USVs. The error system can be decomposed into the cascading system shown in (22) and (23):

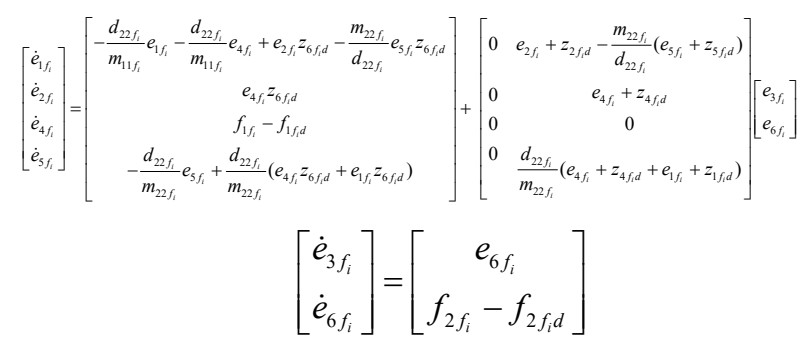

\section{DESIGN OF A CONTROL LAW FOR $f_{1 f_{i}}$}

Based on the system of equations in (22), we first take the system in (24):

$$
\left[\begin{array}{c}
\dot{e}_{1 f_{i}} \\
\dot{e}_{2 f_{i}} \\
\dot{e}_{4 f_{i}} \\
\dot{e}_{5 f_{i}}
\end{array}\right]=\left[\begin{array}{c}
-d_{22 f_{i}} e_{1 f_{i}} / m_{11 f_{i}}-d_{22 f_{i}} e_{4 f_{i}} / m_{11 f_{i}}+e_{2 f_{i}} z_{6 f_{i} d}-m_{22 f_{i}} e_{5 f_{i}} z_{6 f_{i} d} / d_{22 f_{i}} \\
e_{4 f_{i}} z_{6 f_{f} d} \\
f_{1 f_{i}}-f_{1 f_{i} d} \\
-d_{22 f_{i}} e_{5 f_{i}} / m_{22 f_{i}}+d_{22 f_{i}}\left(e_{4 f_{i}} z_{6 f_{i} d}+e_{1 f_{i}} z_{6 f_{i} d}\right) / m_{22 f_{i}}
\end{array}\right]
$$

We then design the following virtual input variable $\tilde{e}_{4 f_{i}}$ for $e_{4 f_{i}}$ as follows:

$$
\tilde{e}_{4 f_{i}}=k_{1 f_{i}} e_{1 f_{i}}-k_{2 f_{i}} z_{6 f_{i} d} e_{2 f_{i}}-k_{3 f_{i}} z_{6 f_{i} d} e_{5 f_{i}}
$$

where $k_{1 f_{i}}, k_{2 f_{i}}$ and $k_{3 f_{i}}$ are all positive definite parameters. 
We define the virtual input variable error between $\tilde{e}_{4 f_{i}}$ and $e_{4 f_{i}}$ as $\Delta_{f_{i}}:$

$$
\Delta_{f_{i}}=e_{4 f_{i}}-\tilde{e}_{4 f_{i}}
$$

Differentiating the error variable in (26) yields:

$\dot{\Delta}_{f i}=\dot{e}_{4 f_{i}}-\dot{\tilde{e}}_{4 f_{i}}=f_{1 f_{i}}-f_{1 f f d}-\left(k_{1 j i} \dot{e}_{1 f_{i}}-k_{2 f i} \dot{z}_{6 f d} e_{2 f_{i}}-k_{2 f_{i}} z_{6 f f d} \dot{e}_{i 2}-k_{3 f_{i}} \dot{z}_{6 f d d} e_{5 f_{i}}-k_{3 f_{i}} z_{6 f d} \dot{e}_{5 f_{i}}\right)$

Combining expressions (24), (25), (26) and (27) gives the following new error system:

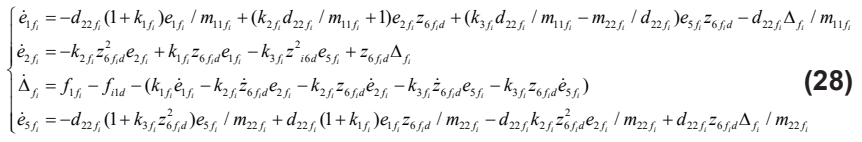

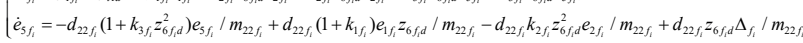

A candidate Lyapunov function for the system in (28) could be designed as follows:

$$
V_{1 f_{i}}=\frac{1}{2} \lambda_{1 f_{i}} e_{1 f_{i}}^{2}+\frac{1}{2} \lambda_{2 f_{i}} e_{2 f_{i}}^{2}+\frac{1}{2} \Delta_{f_{i}}^{2}+\frac{1}{2} \lambda_{3 f_{i}} e_{5 f_{i}}^{2}
$$

where $\lambda_{1 f_{i}}, \lambda_{2 f_{i}}$ and $\lambda_{3 f_{i}}$ are all positive definite parameters. Differentiation of the Lyapunov function in (29) gives:

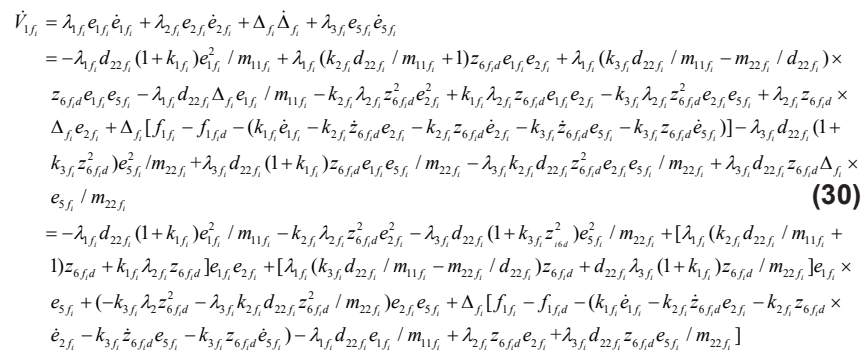

$=-\lambda_{1 f_{i}} d_{22 f_{i}}\left(1+k_{1 f_{i}}\right) e_{1 f_{i}}^{2} / m_{11 f_{i}}-k_{2 f_{i}} \lambda_{2 f_{i}} z_{6 f_{f} d}^{2} e_{2 f_{i}}^{2}-\lambda_{3 f_{i}} d_{22 f_{i}}\left(1+k_{3 f_{i}} z_{i b d}^{2}\right) e_{5 f_{i}}^{2} / m_{22 f_{i}}+\left[\lambda_{1 f_{i}}\left(k_{2 f_{i}} d_{22 f_{i}} / m_{11 f_{i}}+\right.\right.$

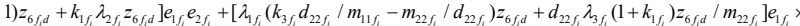
$e_{5 f_{i}}+\left(-k_{3 f_{i}} \lambda_{2} z_{6 f f_{d}}^{2}-\lambda_{3 f_{i}} k_{2 f_{i}} d_{22 f_{i}} z_{6 f f d}^{2} / m_{22 f_{i}}\right) e_{2 f_{i}} e_{5 f_{i}}+\Delta_{f_{i}}\left[f_{1 f_{i}}-f_{1 f_{f d}}-\left(k_{1 f_{i}} \dot{e}_{1 f_{i}}-k_{2 f_{i}} \dot{z}_{6 f f d} e_{2 f_{i}}-k_{2 f_{i}} z_{6 f f_{d}} \times\right.\right.$ $\left.\left.\dot{e}_{2 f_{i}}-k_{3 f_{i}} \dot{z}_{6 f d} e_{5 f_{i}}-k_{3 f_{i}} z_{6 f d} \dot{e}_{5 f_{i}}\right)-\lambda_{1 f_{i}} d_{22 f_{i}} e_{1 f_{i}} / m_{11 f_{i}}+\lambda_{2 f_{i}} z_{6 f d} e_{2 f_{i}}+\lambda_{3 f_{i}} d_{22 f_{i}} z_{6 f d} e_{5 f_{i}} / m_{22 f_{i}}\right]$

According to the expression in (30), a control law for $f_{1 f_{i}}$ can be proposed as follows:

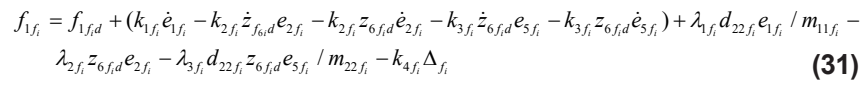

where $k_{4 f_{i}}$ are all positive definite parameters and $f_{1 f_{i} d}$ is as shown in (19).

\section{DESIGN OF A CONTROL LAW FOR $f_{2 f_{i}}$}

According to the system of equations in (23), the virtual input variable $\tilde{e}_{6 f_{i}}$ for $e_{6 f_{i}}$ can be designed as follows:

$$
\tilde{e}_{6 f_{i}}=-k_{5 f_{i}} e_{3 f_{i}}
$$

where $k_{5 f_{i}}$ are all positive definite parameters. We define the virtual input variable error between $\tilde{e}_{6 f_{i}}$ and $e_{6 f_{i}}$ as $\Theta_{f:}$ :

$$
\Theta_{f_{i}}=e_{6 f_{i}}-\tilde{e}_{6 f_{i}}
$$

Differentiation of the error variable in (33) yields:

$$
\dot{\Theta}_{f_{i}}=\dot{e}_{6 f_{i}}-\dot{\tilde{e}}_{6 f_{i}}=f_{2 f_{i}}-f_{2 f_{i} d}-\dot{\tilde{e}}_{6 f_{i}}
$$

Combining expressions (23), (32), (33) and (34) gives the following new error system:

$$
\left\{\begin{array}{l}
\dot{e}_{3 f_{i}}=e_{e 6 f_{i}}-k_{5 f_{i}} e_{3 f_{i}} \\
\dot{\Theta}_{f_{i}}=f_{2 f_{i}}-f_{2 f_{i} d}-\dot{\tilde{e}}_{6 f_{i}}
\end{array}\right.
$$

A candidate Lyapunov function for the system in (35) could be designed as follows:

$$
V_{2 f_{i}}=\frac{1}{2} k_{6 f_{i}} e_{3 f_{i}}^{2}+\frac{1}{2} \Theta_{f_{i}}^{2}
$$

where $k_{6 f_{i}}$ are all positive definite parameters. Differentiation of the Lyapunov function in (36) leads to:

$$
\begin{aligned}
\dot{V}_{2 f_{i}} & =k_{6 f_{i}} e_{3 f_{i}} \dot{e}_{3 f_{i}}+\Theta_{f_{i}} \dot{\Theta}_{f_{i}} \\
& =k_{6 f_{i}} e_{3 f_{i}}\left(\Theta_{f_{i}}-k_{5 f_{i}} e_{3 f_{i}}\right)+\Theta_{f_{i}}\left(f_{2 f_{i}}-f_{2 f_{i} d}-\dot{\tilde{e}}_{6 f_{i}}\right) \\
& =-k_{5 f_{i}} k_{6 f_{i}} e_{3 f_{i}}^{2}+\Theta_{f_{i}}\left(f_{2 f_{i}}-f_{2 f_{i} d}-\dot{\tilde{e}}_{6 f_{i}}+k_{6 f_{i}} e_{3 f_{i}}\right)
\end{aligned}
$$

Based on the expression for $\dot{V}_{2 f_{i}}$ in (37), a control law for $f_{2 f_{i}}$ can be proposed as follows:

$$
\begin{aligned}
f_{2 f_{i}} & =-k_{7 f_{i}} \Theta_{f_{i}}+f_{2 f_{i} d}+\dot{\tilde{e}}_{6 f_{i}}-k_{6 f_{i}} e_{3 f_{i}} \\
& =-k_{7 f_{i}}\left(\Theta_{f_{i}}+k_{5 f_{i}} e_{3 f_{i}}\right)-k_{5 f_{i}} \dot{e}_{3 f_{i}}-k_{6 f_{i}} e_{3 f_{i}}+f_{2 f_{i} d} \\
& =-k_{7 f_{i}} \Theta_{f_{i}}-\left(k_{6 f_{i}}+k_{7 f_{i}} k_{5 f_{i}}\right) e_{3 f_{i}}-k_{5 f_{i}} \dot{e}_{3 f_{i}}+f_{2 f_{i} d}
\end{aligned}
$$

where $k_{7 f_{i}}$ are all positive definite parameters and $f_{2 f_{i d}}$ is as shown in (19).

\section{STABILITY ANALYSIS}

\section{A. STABILITY THEORIES}

In order to better describe the stability analysis process of the control algorithm presented here, the following two classic stability analysis theories are applied.

Theorem 1[28] Consider the system: 


$$
\left\{\begin{array}{l}
\dot{x}=f_{1}(t, x)+g(t, x, y) y \\
\dot{y}=f_{2}(t, y)
\end{array}\right.
$$

where $x \in I R^{n}, y \in I R^{m}, f_{1}(t, x)$ is continuously differentiable with respect to $(t, x)$ and $f_{2}(t, y), g(t, x, y)$ are continuous in their arguments and are locally Lipschitz in $y$ and $(x, y)$ respectively. We can view the system in (39) as:

$$
\dot{x}=f_{1}(t, x)
$$

which is perturbed by the output of the system:

$$
\dot{y}=f_{2}(t, y)
$$

The cascaded system in (39) is globally uniformly asymptotically stable (GUAS) if the following three assumptions hold:

Assumption 1: The system (40) is GUAS and there exists a continuously differentiable function $V(t, x): I R \geq 0 \times I R^{n} \rightarrow I R$ that satisfies:

$$
\left\{\begin{array}{l}
W(x) \leq V(t, x) \\
\frac{\partial V}{\partial t}+\frac{\partial V}{\partial x} \cdot f_{1}(t, x) \leq 0, \forall\|x\| \geq \eta \\
\left\|\frac{\partial V}{\partial x}\right\|\|x\| \leq c V(t, x), \forall\|x\| \geq \eta
\end{array}\right.
$$

where $W(x)$ is a positive definite proper function and $c>0$ and $\eta>0$ are constants.

Assumption 2: For all $t>t_{0}$, the function $g(t, x, y)$ satisfies

$$
\|g(t, x, y)\| \leq \theta_{1}(\|y\|)+\theta_{2}(\|y\|)\|x\|
$$

where $\theta_{1}, \theta_{2}: I R_{\geq 0} \rightarrow I R_{\geq 0}$ are continuous functions.

Assumption 3: The system (41) is GUAS and for all $t_{0} \geq 0$,

$$
\int_{t_{0}}^{\infty}\left\|y\left(t, t_{0}, y\left(t_{0}\right)\right)\right\| d t \leq \kappa\left(\left\|y\left(t_{0}\right)\right\|\right)
$$

where the function $\kappa(\square)$ is a class $\kappa$ function.

Theorem 2[29] Consider the nonautonomous system

$$
\dot{x}=f(t, x)
$$

where $f:[0, \infty) \times D \rightarrow R^{n}$ is piecewise continuous in $t$ and is locally Lipschitz in $x$ on $[0, \infty) \times D$, and $D \subset R^{n}$ is a domain that contains the origin $x=0$. The origin is an equilibrium point for (45) at $t=0$ if $f(t, 0)=0, \forall t \geq 0$. Let $x=0$ be an equilibrium point for (45) and let $D \subset R^{n}$ be a domain containing Let $V:[0, \infty) \times D \rightarrow R$ be a continuously differentiable function such that

$$
\left\{\begin{array}{l}
k_{1}\|x\|^{a} \leq V(t, x) \leq k_{2}\|x\|^{a} \\
\frac{\partial V}{\partial t}+\frac{\partial V}{\partial x} f(t, x) \leq-k_{3}\|x\|^{a}
\end{array} \forall t \geq 0, \forall x \in D\right.
$$

where $k_{1}, k_{2}, k_{3}$ and are positive constants.

Then $x=0$ is exponentially stable. If these assumptions hold globally, then it is globally exponentially stable.

\section{B. STABILITY ANALYSIS}

By combining the expressions (22), (23), (25), (26) and (27), the following system can be derived:

$$
\left\{\begin{array}{l}
\dot{\mathbf{x}}_{f_{i}}=f_{1 f_{i}}\left(t, \mathbf{x}_{f_{i}}\right)+g_{f_{i}}\left(t, \mathbf{x}_{f_{i}}, \mathbf{y}_{f_{i}}\right) \mathbf{y}_{f_{i}} \\
\dot{\mathbf{y}}_{f_{i}}=f_{2 f_{i}}\left(t, \mathbf{y}_{f_{i}}\right)
\end{array}\right.
$$

where $\mathbf{x}_{f_{i}}=\left[e_{1 f_{i}}, e_{2 f_{i}}, \Delta_{f_{i}}, e_{5 f_{i}}\right]^{\mathrm{T}}, \mathbf{y}_{f_{i}}=\left[e_{3 f_{i}}, e_{6 f_{i}}\right]^{\mathrm{T}}$, and

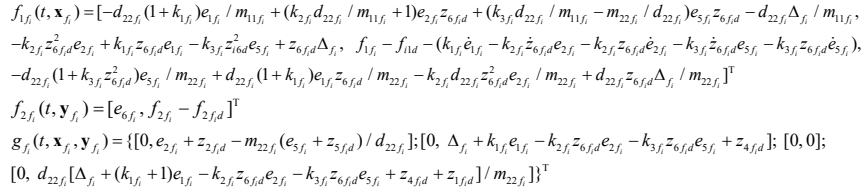

Three steps are taken to prove that the system in (47) satisfies all the conditions of Theory 1, as follows.

Step 1: In order to make the subsequent calculations more straightforward, we define the parameters shown in expression (48):

$$
\left\{\begin{array}{l}
c_{1 f_{i}}=\lambda_{1 f_{i}}\left(k_{2} d_{22 f_{i}} / m_{11 f_{i}}+1\right)+k_{1 f_{i}} \lambda_{2 f_{i}} \\
c_{2 f_{i}}=\lambda_{1 f_{i}} k_{3 f_{i}} d_{22 f_{i}} / m_{11 f_{i}}-\lambda_{3 f_{i}} d_{22 f_{i}}\left(1+k_{1 f_{i}}\right) / m_{22 f_{i}} \\
c_{3 f_{i}}=k_{3 f_{i}} \lambda_{2 f_{i}}-\lambda_{3 f_{i}} k_{2 f_{i}} d_{22 f_{i}} / m_{22 f_{i}} \\
c_{4 f_{i}}=\lambda_{1 f_{i}} m_{22 f_{i}} / d_{22 f_{i}}
\end{array}\right.
$$

By combining the control law in (31) for $f_{1 f_{i}}$ with the Lyapunov function in (30), we obtain:

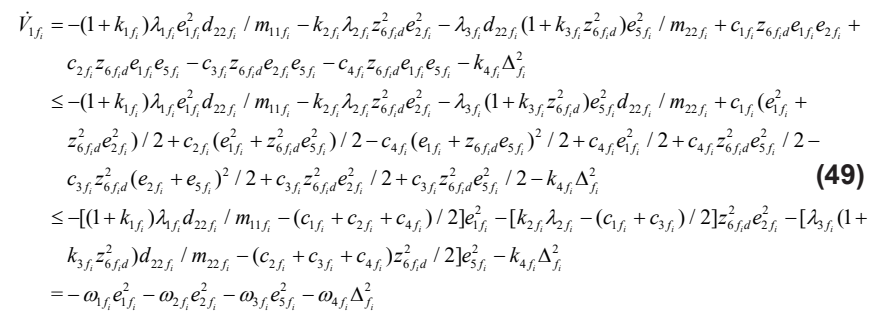


where $\left\{\begin{array}{l}\omega_{1 f_{i}}=\left[\left(1+k_{1 f_{i}}\right) \lambda_{1 f_{i}} d_{22 f_{i}} / m_{11 f_{i}}-\left(c_{1 f_{i}}+c_{2 f_{i}}+c_{4 f_{i}}\right) / 2\right] \\ \omega_{2 f_{i}}=\left[k_{2 f_{i}} \lambda_{2 f_{i}}-\left(c_{1 f_{i}}+c_{3 f_{i}}\right) / 2\right] z_{6 f_{i} d}^{2} \\ \omega_{3 f_{i}}=\left[\lambda_{3 f_{i}} d_{22 f_{i}}\left(1+k_{3 f_{i}} z_{6 f_{i} d}^{2}\right) / m_{22 f_{i}}-\left(c_{2 f_{i}}+c_{3 f_{i}}+c_{4 f_{i}}\right) z_{6 f_{i} d}^{2} / 2\right] \\ \omega_{4 f_{i}}=k_{4 f_{i}}\end{array}\right.$

If parameters such as $k_{1 f_{i}}, k_{2 f_{i}}, k_{3 f_{i}}, k_{4 f_{i}}, \lambda_{1 f_{i}}, \lambda_{2 f_{i}}$ and $\lambda_{3 f_{i}}$ are selected appropriately, then the parameters $\omega_{1 f_{i}}, \omega_{2 f_{i}}, \omega_{3 f_{i}}$ and $\omega_{4 f_{i}}$ can be guaranteed to be non-negative. The following results can then also be derived:

$\left\{\begin{array}{l}V_{f_{i}}\left(t, \mathbf{x}_{f_{i}}\right)=V_{1 f_{i}} \geq \min \left\{\lambda_{1 f_{i}}, \lambda_{2 f_{i}}, 1, \lambda_{3 f_{i}}\right\}\left\|\mathbf{x}_{f_{i}}\right\|^{2} \geq \frac{1}{2} \min \left\{\lambda_{1 f_{i}}, \lambda_{2 f_{i}}, 1, \lambda_{3 f_{i}}\right\}\left\|\mathbf{x}_{f_{i}}\right\|^{2}=W_{f_{i}}\left(\mathbf{x}_{f_{i}}\right) \\ \dot{V}_{1 f_{i}}=\frac{\partial V_{1 f_{i}}}{\partial t}+\frac{\partial V_{1 f_{i}}}{\partial \mathbf{x}_{f_{i}}} f_{1 f_{i}}\left(t, \mathbf{x}_{f_{i}}\right) \leq 0,\left\|\mathbf{x}_{f_{i}}\right\| \geq \eta_{f_{i}}\end{array}\right.$

where $W_{f_{i}}\left(\mathbf{x}_{f_{i}}\right)$ are positive definite proper functions and $\eta_{f_{i}}>0$ are constants. Since

$$
\left\|\frac{\partial V_{1 f_{i}}}{\partial \mathbf{x}}\right\| \leq \max \left\{\lambda_{1 f_{i}}, \lambda_{2 f_{i}}, 1, \lambda_{3 f_{i}}\right\}\left\|\mathbf{x}_{f_{i}}\right\|
$$

the following expression can be obtained:

$\left\|\frac{\partial V_{1 f_{i}}}{\partial \mathbf{x}_{f_{i}}}\right\|\left\|\mathbf{x}_{f_{i}}\right\| \leq \max \left\{\lambda_{1 f_{i}}, \lambda_{2 f_{i}}, 1, \lambda_{3 f_{i}}\right\}\left\|\mathbf{x}_{f_{i}}\right\|^{2} \leq \frac{2 \max \left\{\lambda_{1 f_{i}}, \lambda_{2 f_{i}}, 1, \lambda_{3 f_{i}}\right\}}{\min \left\{\lambda_{1 f_{i}}, \lambda_{2 f_{i}}, 1, \lambda_{3 f_{i}}\right\}} V_{1 f_{i}}=c_{f_{i}} V_{f_{i}}\left(t, \mathbf{x}_{f_{i}}\right)$

where $c_{f_{i}}=2 \max \left\{\lambda_{1 f_{i}}, \lambda_{2 f_{i}}, 1, \lambda_{3 f_{i}}\right\} / \min \left\{\lambda_{1 f_{i}}, \lambda_{2 f_{i}}, 1, \lambda_{3 f_{i}}\right\}$ are positive constants. Thus, Assumption 1 of Theory 1 has been satisfied.

Step 2: In this step, the correlation function $g_{f_{i}}\left(t, \mathbf{x}_{f_{i}}, \mathbf{y}_{f_{i}}\right)$ in (47) is analysed. This can be expressed as follows:

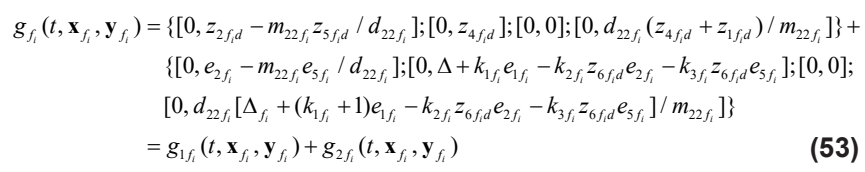

where,

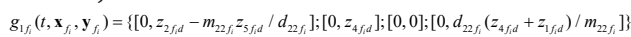

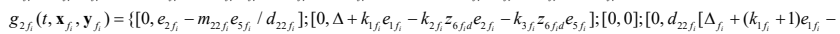
$\left.\left.k_{2 f i} z_{6 f i d} e_{2 f i}-k_{3 f i} z_{6 f i d} e_{5 i}\right] / m_{22 f i}\right]$

From norm theory, we can show that:

$$
\left\|g_{f_{i}}\left(t, \mathbf{x}_{f_{i}}, \mathbf{y}_{f_{i}}\right)\right\| \leq\left\|g_{1 f_{i}}\left(t, \mathbf{x}_{f_{i}}, \mathbf{y}_{f_{i}}\right)\right\|+\left\|g_{2 f_{i}}\left(t, \mathbf{x}_{f_{i}}, \mathbf{y}_{f_{i}}\right)\right\|
$$

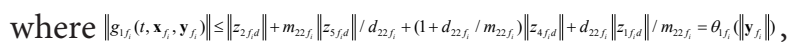

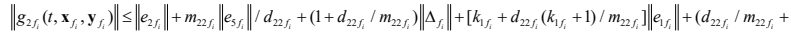

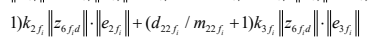

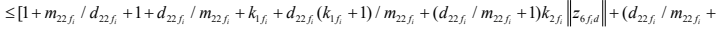

1) $k_{3, f}\left\|z_{6 f d}|| \mid \mathbf{x}_{f i f}\right\|$

$=\theta_{2 f}\left|\left\|y_{f}\right\|\right|\left\|x_{f}\right\|$

and $\left.\left.\theta_{1}\right\}_{i}\right\rangle \mathbf{y}_{f_{i}}\left\|, \theta_{2 f_{i}}\right\| \mathbf{y}_{f_{i}}\|\| \mathbf{x}_{f_{f}} \|: \mathbf{R}(\geq 0) \rightarrow \mathbf{R}(\geq 0)$ are continuous functions. The following expression therefore holds:

$\left.\left\|g_{f_{i}}\left(t, \mathbf{x}_{f_{i}}, \mathbf{y}_{f_{i}}\right)\right\| \leq \theta_{1 f_{i}}\right)\left\|\mathbf{y}_{f_{i}}\right\|+\theta_{2 f_{i}}\left\|\mathbf{y}_{f_{i}}\right\|\left\|\mathbf{x}_{f_{i}}\right\|$ which satisfies Assumption 2 of Theory 1.

Step 3: Combining the control law in (38) for $f_{2 f_{i}}$ with the Lyapunov function in (37) leads to:

$\dot{V}_{2 f_{i}}=-k_{5 f_{i}} k_{6 f_{i}} e_{3 f_{i}}^{2}-k_{7 f_{i}} \Theta_{f_{i}}^{2} \leq-\min \left\{k_{5 f_{i}}, k_{6 f_{i}}, k_{7 f_{i}}\right\}\left\|\left[e_{3 f_{i}}, \Theta_{f_{i}}\right]\right\|^{2}=-\sigma_{3 f_{i}}\left\|\left[e_{3 f_{i}}, \Theta_{f_{i}}\right]\right\|^{2}$

and

$\min \left\{k_{6 f_{i}} / 2,1 / 2\right\}\left\|\left[e_{3 f_{i}}, \Theta_{f_{i}}\right]\right\|^{2} \leq V_{2 f_{i}} \leq \max \left\{k_{6 f_{i}} / 2,1 / 2\right\}\left\|\left[e_{3 f_{i}}, \Theta_{f_{i}}\right]\right\|^{2}$

where $\sigma_{1 f_{i}}=\min \left\{k_{6 f_{i}} / 2,1 / 2\right\}, \sigma_{2 f_{i}}=\max \left\{k_{6 f_{i}} / 2,1 / 2\right\}$.

According to Theory $2,\left[e_{3 f_{i}}, \Theta_{f_{i}}\right]=\mathbf{0}$ is globally exponentially stable. If we combine the expressions in (32) and (33), we can shown that $\left[e_{3 f_{i},}, e_{6 f_{i}}\right]=\mathbf{0}$ is globally exponentially stable.

$$
\left\|\mathbf{y}_{f_{i}}\left(t, t_{0}, \mathbf{y}_{f_{i}}\left(t_{0}\right)\right)\right\| \leq k_{f_{i}}\left\|\mathbf{y}_{f_{i}}\left(t_{0}\right)\right\| e^{-\lambda_{f_{i}}\left(t-t_{0}\right)}, \forall\left\|\mathbf{y}_{f_{i}}\left(t_{0}\right)\right\| \leq \delta_{f_{i}}
$$

where $k_{f_{i}}, \lambda_{f_{i}}$ and $\delta_{f_{i}}$ are positive constants and $\delta_{f_{i}}$ can be arbitrarily small. Then

$\int_{t_{0}}^{\infty}\left\|\mathbf{y}_{f_{i}}\left(t, t_{0}, \mathbf{y}_{f_{i}}\left(t_{0}\right)\right)\right\| \leq \int_{t_{0}}^{\infty} k_{f_{i}}\left\|\mathbf{y}_{f_{i}}\left(t_{0}\right)\right\| e^{-\lambda_{f_{i}}\left(t-t_{0}\right)}=k_{f_{i}}\left\|\mathbf{y}_{f_{i}}\left(t_{0}\right)\right\| / \lambda_{f_{i}}=\kappa_{f_{i}}\left(\left\|\mathbf{y}_{f_{i}}\left(t_{0}\right)\right\|\right)$

where the function $\kappa_{f}(\square)$ is a class $\kappa$ function.

Hence, the system described in (47) satisfies all the conditions of Theory 1 . The system in (47) is GUAS, and the variables $\mathbf{x}_{f_{i}}$ and $\mathbf{y}_{f_{i}}$ are GUAS to $\mathbf{0}$. By combining expressions (25) and (26), we see that $\left[e_{1 f_{i}}, e_{2 f_{i}}, e_{3 f_{i}}, e_{4 f_{i}}, e_{5 f_{i}}, e_{6 f_{i}}\right]=\mathbf{0}$ is GUAS. Based on the tracking error system described in (21), stable cooperative formation control of the USVs can be realised using the control law expressed in (31) and (38).

\section{SIMULATION EXPERIMENT}

Several simulation experiments were carried out to verify the effectiveness and reliability of our cooperative formation control algorithm. The mathematical model for the USVs is taken from [30], and the parameter values are shown in Table 1 .

Tab. 1. Model parameters

\begin{tabular}{|c|c|c|c|}
\hline Parameters & Value & Parameters & Value \\
\hline$m_{11}$ & 25.8 & $d_{11}$ & 12 \\
\hline$m_{22}$ & 33.8 & 22 & 17 \\
\hline$m_{33}$ & 2.76 & $d_{33}$ & 0 \\
\hline
\end{tabular}

The trajectory of the leader USV is generated by the following expression, which can be used to design different forms of leader trajectory. 


$$
\left\{\begin{array}{l}
\dot{x}_{\mathrm{d}}=u_{d} \cos \varphi_{d}-v_{d} \sin \varphi_{d} \\
\dot{y}_{d}=u_{d} \sin \varphi_{d}+v_{d} \cos \varphi_{d} \\
\dot{\varphi}_{d}=r_{d} \\
\dot{v}_{d}=-m_{11} u_{d} r_{d} / m_{22}-d_{22} v_{d} / m_{22}
\end{array}\right.
$$

Three USVs with same model parameters (a leader USV and two follower USVs) were used in the simulation experiment, and three kinds of trajectory (a straight line, a circular trajectory and a general S-shaped trajectory) were designed to demonstrate the performance of our control algorithm. In order to verify the robustness and stability of the proposed algorithm, a stable disturbance and a sinusoidal disturbance were added in the longitudinal and steering control directions. Disturbances of $d_{u}=2+0.1 \sin (0.2 t)$ in the longitudinal direction and $d_{r}=1+0.1 \sin (0.5 t)$ in the heading control direction were also added.

Since there are numerous control parameters that need to be set for the control system, several basic principles were followed: (a) all the parameters should be positive, since they are all defined as positive; (b) the parameters for each follower should be the same except for $k_{1 f_{i}}$, since different speeds are needed for different followers; (c) $k_{6 f_{i}}$ should be small, since a higher value of $k_{6 f_{i}}$ means a lower convergence speed, as can be seen from (56) and (57); (d) $\lambda_{n f}$ can be chosen arbitrarily, as this has a minor impact on the system.

Case 1: A straight line trajectory is designed for the leader USV, and in order to ensure a cooperative formation, the positional information of the follower USVs relative to the leader USV is determined based on the parameters in Table 2. The initial states of the USVs and the control parameters are also shown in Table 2, and the simulation results are presented in Figs. 3 and 4.

Tab. 2. Initial states and control parameters

\begin{tabular}{|c|c|c|c|c|c|}
\hline Symbol & Value & Symbol & Value & Symbol & Value \\
\hline$u_{l d}$ & $10 \mathrm{~m} / \mathrm{s}$ & $L_{1}$ & $5 \mathrm{~m}$ & $L_{2}$ & $5 \mathrm{~m}$ \\
\hline$r_{l d}$ & 0 & $\theta_{1}$ & $\pi / 2$ & $\theta_{2}$ & $-\pi / 2$ \\
\hline$x_{l}(0)$ & $15 \mathrm{~m}$ & $x_{f_{1}}(0)$ & $9 \mathrm{~m}$ & $x_{f_{2}}(0)$ & $20 \mathrm{~m}$ \\
\hline$y_{l}(0)$ & $-6 \mathrm{~m}$ & $y_{f_{1}}(0)$ & $8 \mathrm{~m}$ & $y_{f_{2}}(0)$ & $-7 \mathrm{~m}$ \\
\hline$\varphi_{l}(0)$ & $0.7 \mathrm{rad}$ & $\varphi_{f_{1}}(0)$ & $0.3 \mathrm{rad}$ & $\varphi_{f_{2}}(0)$ & $0.3 \mathrm{rad}$ \\
\hline$u_{1}(0)$ & $0.5 \mathrm{~m} / \mathrm{s}$ & $u_{f_{1}}(0)$ & $0.5 \mathrm{~m} / \mathrm{s}$ & $u_{f_{2}}(0)$ & $0.5 \mathrm{~m} / \mathrm{s}$ \\
\hline$v_{1}(0)$ & $0.5 \mathrm{~m} / \mathrm{s}$ & $v_{f_{1}}(0)$ & $0.5 \mathrm{~m} / \mathrm{s}$ & $v_{f_{2}}(0)$ & $0.5 \mathrm{~m} / \mathrm{s}$ \\
\hline$r_{1}(0)$ & 0 & $r_{f_{1}}(0)$ & 0 & $r_{f_{2}}(0)$ & 0 \\
\hline$k_{1 l}$ & 2.3 & $k_{1 f_{1}}$ & 1.6 & $k_{1 f_{2}}$ & 0.03 \\
\hline$k_{2 l}$ & 5 & $k_{2 f_{1}}$ & 15 & $k_{2 f_{2}}$ & 15 \\
\hline$k_{3 l}$ & 5 & $k_{3 f_{1}}$ & 15 & $k_{3 f_{2}}$ & 15 \\
\hline$k_{4 l}$ & 3 & $k_{4 f_{1}}$ & 15 & $k_{4 f_{2}}$ & 13 \\
\hline$k_{5 l}$ & 25 & $k_{5 f_{1}}$ & 20 & $k_{5 f_{2}}$ & 20 \\
\hline
\end{tabular}

\begin{tabular}{|c|c|c|c|c|c|}
\hline Symbol & Value & Symbol & Value & Symbol & Value \\
\hline$k_{6 l}$ & 1 & $k_{6 f_{1}}$ & 0.001 & $k_{6 f_{2}}$ & 5 \\
\hline$k_{7 l}$ & 1 & $k_{7 f_{1}}$ & 1 & $k_{7 f_{2}}$ & 1 \\
\hline$\lambda_{1 l}$ & 1 & $\lambda_{1 f_{1}}$ & 1 & $\lambda_{1 f_{2}}$ & 1 \\
\hline$\lambda_{2 l}$ & 1 & $\lambda_{2 f_{1}}$ & 1 & $\lambda_{2 f_{2}}$ & 1 \\
\hline$\lambda_{3 l}$ & 1 & $\lambda_{3 f_{1}}$ & 1 & $\lambda_{3 f_{2}}$ & 1 \\
\hline
\end{tabular}

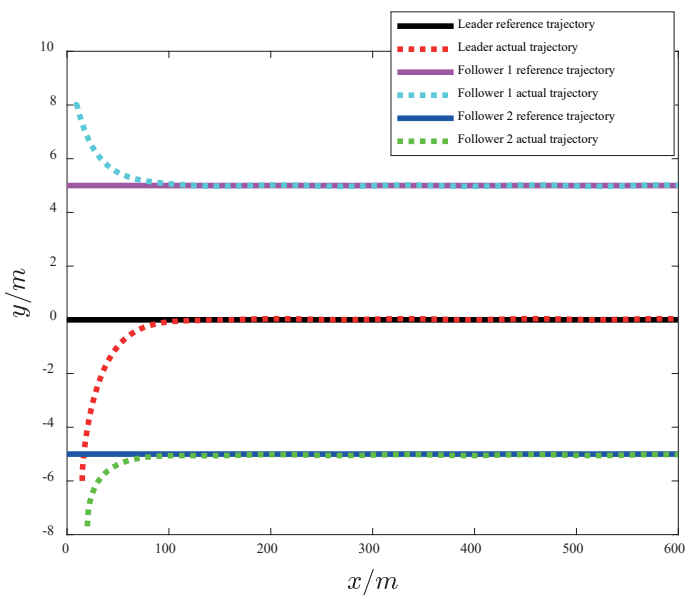

Fig. 3. Results from our cooperative formation control algorithm for a straight line trajectory

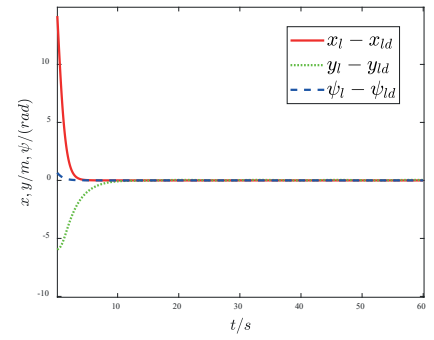

(a) Convergence curve for the position and heading angle of the leader USV

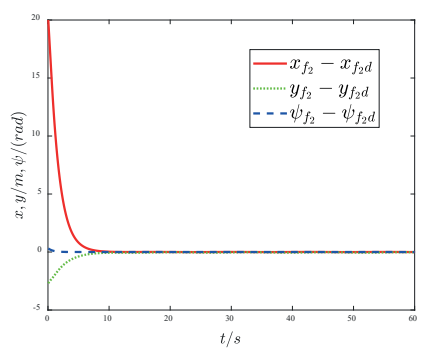

(c) Convergence curve for the position and heading angle of the Follower 2 USV

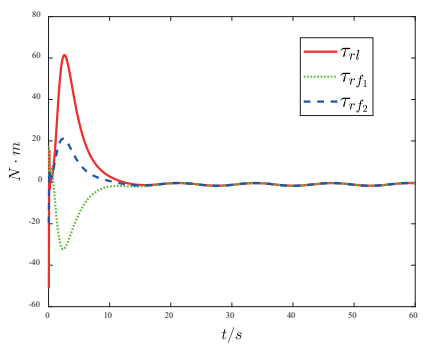

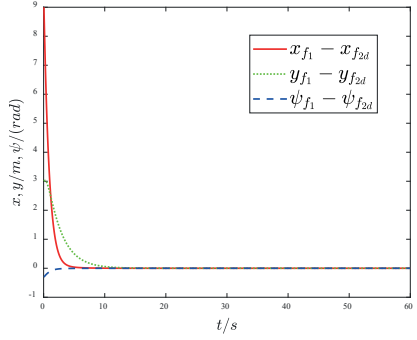

(b) Convergence curve for the position and heading angle of the Follower 1 USV

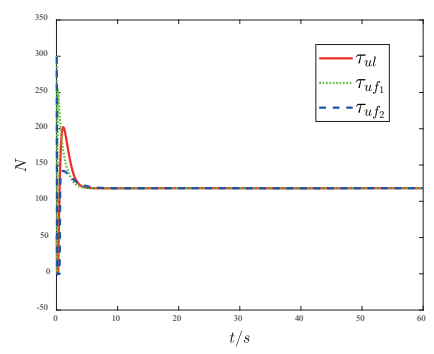

(d) Surge control forces for the three USVS

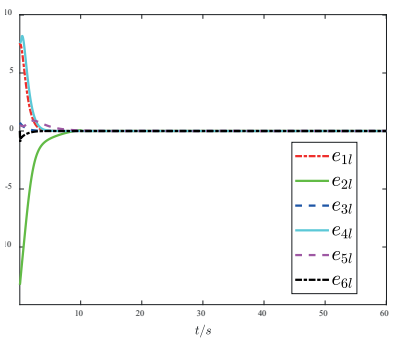


(e) Yaw control torque for the three USVS

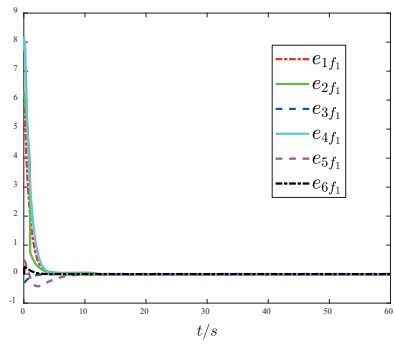

(g) Convergence of intermediate states for the Follower 1 USV (f) Convergence of intermediate states for the Leader USV

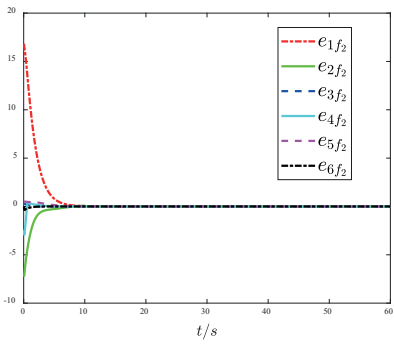

(h) Convergence of intermediate states for the Follower 2 USV

Fig. 4 Detailed error convergence curves and control forces for Case 1

Case 2: A circular trajectory was designed for the leader USV, and in order to ensure a cooperative formation, the positional information for the follower USVs relative to the leader was designed as shown by the initial states in Table 3. The initial states of the USVs are also shown in Table 3 , and the results of the simulation are presented in Figs. 5 and 6. Tab. 3. Initial states for Case 2

\begin{tabular}{|c|c|c|c|c|c|}
\hline Symbol & Value & Symbol & Value & Symbol & Value \\
\hline$u_{l d}$ & $10 \mathrm{~m} / \mathrm{s}$ & $L_{1}$ & $20 \mathrm{~m}$ & $L_{2}$ & $20 \mathrm{~m}$ \\
\hline$r_{l d}$ & $0.1 \mathrm{rad} / \mathrm{s}$ & $\theta_{1}$ & $\pi / 2$ & $\theta_{2}$ & $-\pi / 2$ \\
\hline$x_{l}(0)$ & $15 \mathrm{~m}$ & $x_{f_{1}}(0)$ & $9 \mathrm{~m}$ & $x_{f_{2}}(0)$ & $20 \mathrm{~m}$ \\
\hline$y_{l}(0)$ & $-6 \mathrm{~m}$ & $y_{f_{1}}(0)$ & $15 \mathrm{~m}$ & $y_{f_{2}}(0)$ & $-25 \mathrm{~m}$ \\
\hline$\varphi_{l}(0)$ & $0.7 \mathrm{rad}$ & $\varphi_{f_{1}}(0)$ & $0.3 \mathrm{rad}$ & $(0)$ & $0.7 \mathrm{rad}$ \\
\hline$u_{1}(0)$ & 0 & $u_{f_{1}}(0)$ & 0 & $u_{f_{2}}(0)$ & 0 \\
\hline$v_{1}(0)$ & 0 & $v_{f_{1}}(0)$ & 0 & $v_{f_{2}}(0)$ & 0 \\
\hline$r_{1}(0)$ & 0 & $r_{f_{1}}(0)$ & 0 & $r_{f_{2}}(0)$ & 0 \\
\hline
\end{tabular}

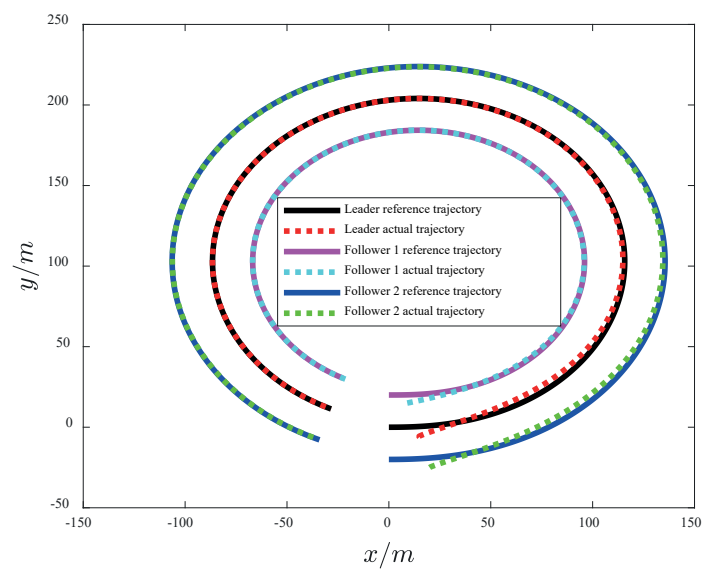

Fig. 5. Results of our cooperative formation control algorithm for a circular trajectory

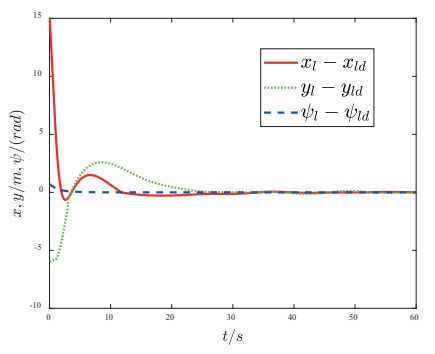

(a) Convergence curve for the position and heading angle of the leader USV

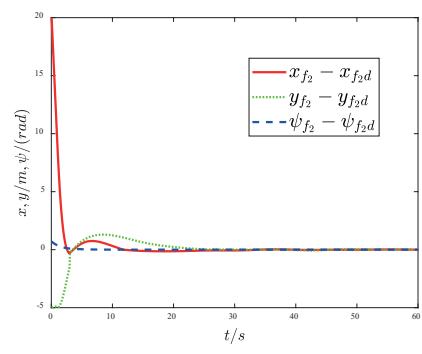

(c) Convergence curve for the position and heading angle of the Follower 2 USV

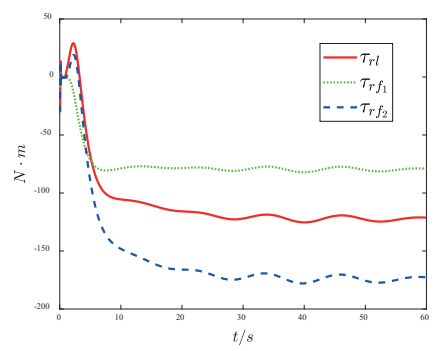

(e) Yaw control torque for the three USVS

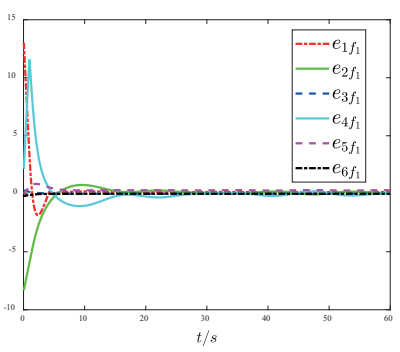

(g) Convergence of intermediate states for the Follower 1 USV;

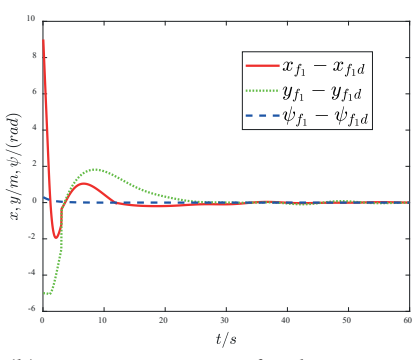

(b) Convergence curve for the position and heading angle of the Follower 1 USV

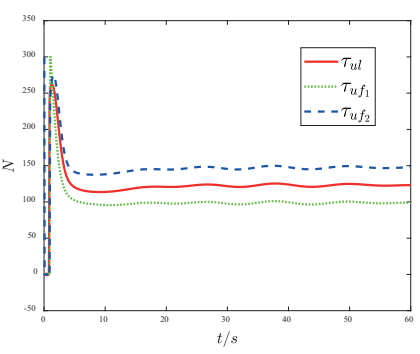

(d) Surge control forces for the three USVs

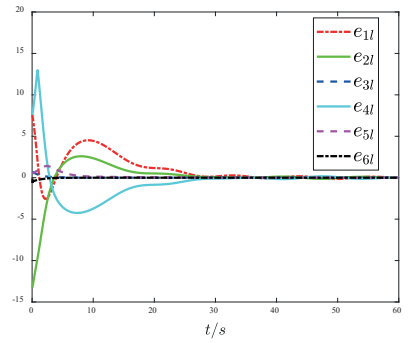

(f) Convergence of intermediate states for the Leader USV;

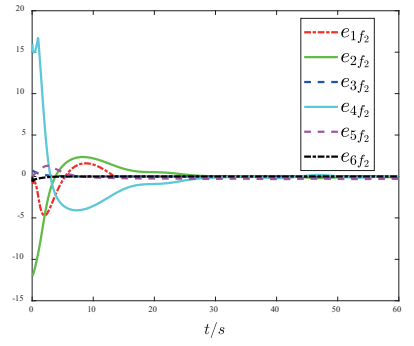

(h) Convergence of intermediate states for the Follower 2 USV
Fig. 6. Detailed error convergence curves and control forces for Case 2

Case 3: A general S-shaped trajectory was designed for the leader USV by setting $u_{l d}=10 \mathrm{~m} / \mathrm{s}, \quad r_{l d}=0.1 \mathrm{rad} / \mathrm{s}, t \leq 30$ and $r_{l d}=-0.05 \mathrm{rad} / s, t>30$. In order to ensure a cooperative formation, the positional information for the follower USVs relative to the leader USV was designed based on the parameters shown in Table 4. The initial states of the USVs are also shown in Table 4, and the results of the simulation are presented in Figs. 7 and 8 . 
Tab. 4. Initial states for case 3

\begin{tabular}{|c|c|c|c|c|c|}
\hline Symbol & Value & Symbol & Value & Symbol & Value \\
\hline$u_{l d}$ & $10 \mathrm{~m} / \mathrm{s}$ & $L_{1}$ & $30 \mathrm{~m}$ & $L_{2}$ & $30 \mathrm{~m}$ \\
\hline$r_{l d}$ & $0.1 /-0.05$ & $\theta_{1}$ & $\pi / 2$ & $\theta_{2}$ & $-\pi / 2$ \\
\hline$x_{l}(0)$ & $15 \mathrm{~m}$ & $x_{f_{1}}(0)$ & 0 & $x_{f_{2}}(0)$ & $20 \mathrm{~m}$ \\
\hline$y_{l}(0)$ & $-6 \mathrm{~m}$ & $y_{f_{1}}(0)$ & $20 \mathrm{~m}$ & $y_{f_{2}}(0)$ & $-45 \mathrm{~m}$ \\
\hline$\varphi_{l}(0)$ & $0.7 \mathrm{rad}$ & $\varphi_{f_{1}}(0)$ & $0.7 \mathrm{rad}$ & $\varphi_{f_{2}}(0)$ & $0.7 \mathrm{rad}$ \\
\hline$u_{1}(0)$ & 0 & $u_{f_{1}}(0)$ & 0 & $u_{f_{2}}(0)$ & 0 \\
\hline$v_{1}(0)$ & 0 & $v_{f_{1}}(0)$ & 0 & $v_{f_{2}}(0)$ & 0 \\
\hline$r_{1}(0)$ & 0 & $r_{f_{1}}(0)$ & 0 & $r_{f_{2}}(0)$ & 0 \\
\hline
\end{tabular}

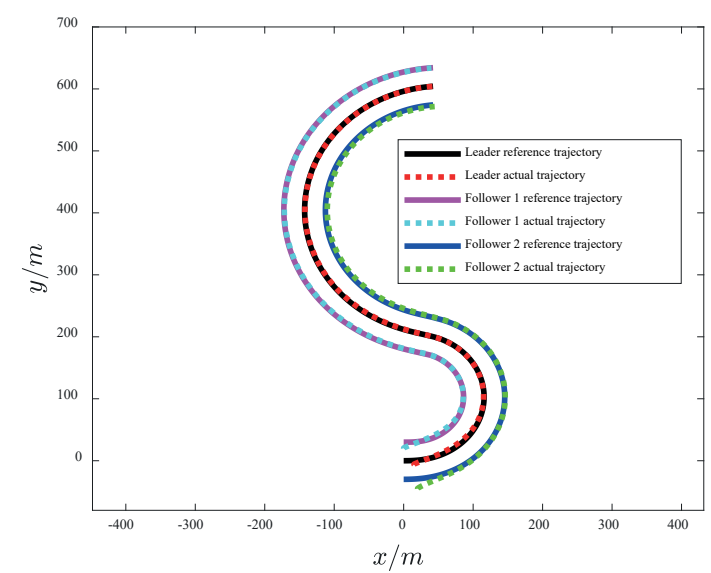

Fig. 7 Results from our cooperative formation control algorithm for a general S-shaped trajectory

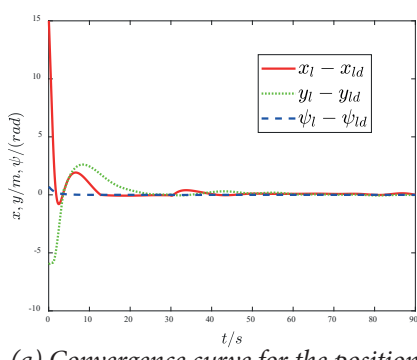

(a) Convergence curve for the position and heading angle of the leader USV

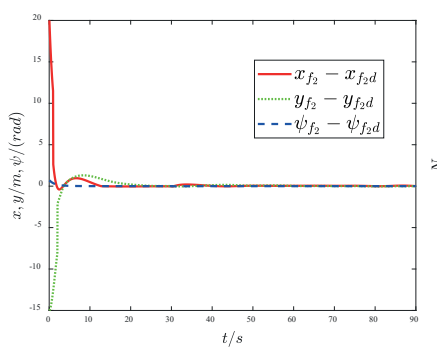

(c) Convergence curve for the position and heading angle of the Follower-2 USV

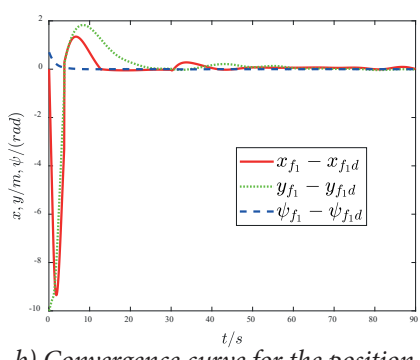

b) Convergence curve for the position and heading angle of the Follower 1 USV

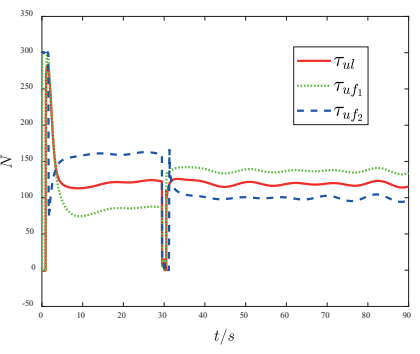

(d) Surge control forces for the three USVs

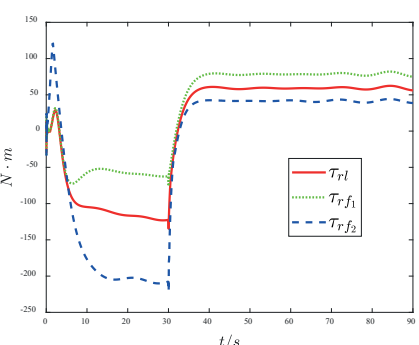

(e) Yaw control torque for the three USVS

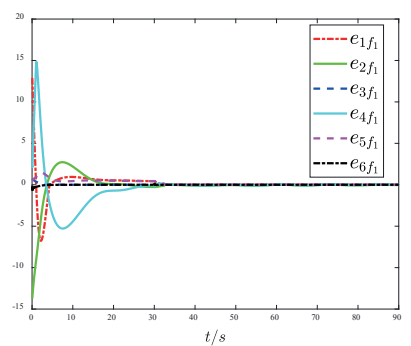

(g) Convergence of intermediate states (h) Convergence of intermediate states for the Follower 1 USV

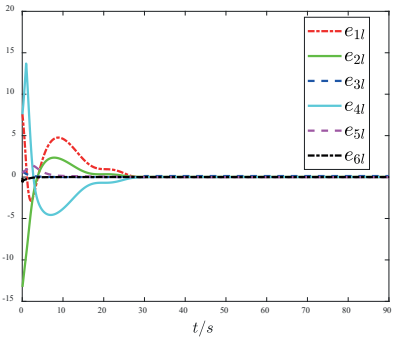

(f) Convergence of intermediate states for the Leader USV

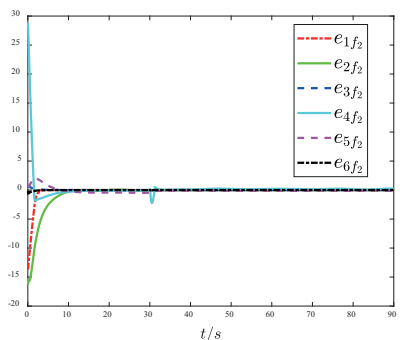

for the Follower 2 USV

Fig. 8 Detailed error convergence curves and control forces of Case 3

The simulation results shown above in Figs. 3 to 8 indicate that the necessary system convergence time for each kind of trajectory tracking processes is within the range 10-30 seconds, and there are no instabilities or divergences after the system has converged. It is therefore obvious that the cooperative formation control algorithm proposed in this paper is stable, reliable and effective. Tracking control was successful for all three kinds of formation trajectories examined here, indicating that the general applicability of our control method is superior to conventional algorithms. From the simulation results presented above, and compared with the results reported in the literature [11] and [22], we can see that the convergence time of the proposed control algorithm is shorter, and the stability of control is better in our simulation results. In addition, the actual tracking trajectory of the USVs is smoother, and our algorithm has a wider range of applications for different kind of trajectories.

In Cases 2 and 3, the control inputs of surge control force and yaw control torque do not converge to zero, since the reference trajectories are curved in these cases, and certain control inputs in terms of both the surge control force and yaw control torque are required to ensure the sailing formation of the USVs. In addition, the actual control inputs are different, since the length of each trajectory is not the same and the necessary velocities are different. In Case 3, a state switch occurs in the middle part of the reference trajectory (that is, a change in the desired angular velocity), meaning that both the surge control force and yaw control torque undergo an abrupt adjustment process, as shown in Figs. 8(d) and (e). From the results for Case 3, we know that in order to change the formation of the USVs, the control inputs for both the surge and yaw vary rapidly due to the fast convergence of the control algorithm; if the desired trajectory is smooth transition designed then this sudden adjustment could be 
avoided. In an actual application, the rate of change could be set to an even value based on the change rate of the propulsion devices of USVs. In addition, for USVs that are not equipped with reversible propellers, the surge control force could also be set to a non-negative value throughout the cooperative formation control process of the underactuated USVs.

\section{CONCLUSION}

A method of cooperative formation control is proposed in this paper for a group of underactuated USVs based on nonlinear backstepping and cascade system theory. A novel description of the problem of cooperative formation control of a group of USVs is presented, and the desired positions and attitude angles of the follower USVs are transformed into intermediate variables that facilitate the design of the controller. A new type of global differential homeomorphism transformation is put forward for the tracking error system of the follower USVs in order to simplify the description of the control system; this makes the complex formation control system easy to analyse and means that it can be decomposed into a cascaded system. In order to improve the stability of the cooperative formation controller, novel intermediate state variables and virtual control laws are devised for use in design process of the control algorithm. By combining the backstepping technique, Lyapunov's direct method and cascade system theory, we develop an improved controller for cooperative formation control of a group of underactuated USVs. We prove that our cooperative formation control algorithm for USVs is GUAS, and a variety of simulation experiments are carried out to verify the validity, stability and reliability of the algorithm, which show that the general applicability of the control method designed is superior to conventional algorithms. Some interference factors such as actual ocean environmental disturbances and uncertainties in the USV models are not fully considered in this paper, and we intend to focus on these in future research work.

\section{ACKNOWLEDGEMENTS}

This research was funded by the National Natural Science Foundation of China (Grant No. 51709214, 51809203, 51879210), Xiangyang Science and Technology Plan Project (Grant No. 2017YL12, 2019ZD03, Hubei Superior and Distinctive Discipline Group of „Mechatronics and Automobiles" ( Grant No. XKQ2021012)

\section{REFERENCES}

1. J. F. Jimenez and J. M. Giron-Sierra, "USV based automatic deployment of booms along quayside mooring ships: Scaled experiments and simulations," Ocean Engineering, vol. 207, pp. 1-12, Jul. 2020. doi:10.1016/j.oceaneng.2020.107438.
2. J. Y. Zhuang, L. Zhang, Z. H. Qin, H. B. Sun, B. Wang, and J. Cao, "Motion control and collision avoidance algorithm for unmanned surface vehicle swarm in practical maritime environment," Polish Maritime Research, vol. 26, no. 1, pp.107-116. doi: 10.2478/pomr-2019-0012.

3. B. C. Shah and S. K. Gupta, "Long-distance path planning for unmanned surface vehicles in complex marine environment," IEEE Journal of Oceanic Engineering, vol. 45, no. 3, pp. 813-830, Jul. 2020. doi:10.1109/JOE.2019.2909508.

4. X. Liang, X. R. Qu, Y. H. Hou, Y. Li, and R. B. Zhang, "Distributed coordinated tracking control of multiple unmanned surface vehicles under complex marine environments," Ocean Engineering, vol. 205, pp. 1-9, Jun. 2020. doi:10.1016/j.oceaneng.2020.107328.

5. H. N. Esfahani and R. Szlapczynski, "Model predictive super-twisting sliding mode control for an autonomous surface vehicle”, Polish Maritime Research, vol. 26, no. 3, pp. 163-171, Sept. 2019. doi: 10.2478/pomr-2019-0057.

6. M. A. Hinostroza, H. T. Xu, and C. G. Soares, "Cooperative operation of autonomous surface vehicles for maintaining formation in complex marine environment," Ocean Engineering, vol. 183, pp. 132-154, Jul. 2019. doi:10.1016/j. oceaneng.2019.04.098.

7. R. V. C. Vid, J. P. V. S. Cunha, and P. B. Garcia-Rosa, "Stabilizing control of an unmanned surface vehicle pushing a floating load ," International Journal of Control, Automation and Systems, vol. 18, pp. 1-10, Jun. 2020. doi:10.1007/s12555-019-0677-1.

8. S. S. Wang and Y. L. Tuo, "Robust trajectory tracking control of underactuated surface vehicle with prescribed performance," Polish Maritime Research, vol. 27, no. 4, pp. 148-156, Dec. 2020. doi: 10.2478/pomr-2020-0075.

9. C. Paliotta, E. Lefeber, K. Y. Pettersen, J. Pinto, M. Costa, and J. T. D. B. Sousa, “Trajectory tracking and path following for underactuated marine vehicles," IEEE Transactions on Control Systems Technology, vol. 27, no. 4, pp. 1423-1437, Jul. 2019. doi:10.1109/TCST.2018.283-4518.

10. J. Han and J. Kim, “Three-dimensional reconstruction of a marine floating structure with an unmanned surface vessel," IEEE Journal of Oceanic Engineering, vol. 44, no. 4, pp. 984-996, Oct. 2019. doi:10.11-09/JOE.2018.2862618.

11. K. Shojaei, "Leader-follower formation control of underactuated autonomous marine surface vehicles with limited torque," Ocean Engineering, vol. 105, pp. 196-205, Jun. 2015. doi:10.1016/j.oceaneng. 2015.06.026.

12. Z. Y. Gao and G. Guo, "Adaptive formation control of autonomous underwater vehicles with model uncertainties," 
Int. J. Adapt. Control Signal Process, vol. 32, pp. 1067-1080, Mar. 2018. doi:10.1002/acs. 2886.

13. J. Ghommam and M. Saad, "Adaptive leader-follower formation control of underactuated surface vessels under asymmetric range and bearing constraints," IEEE Transactions on Control Systems Technology, vol. 67, no. 2, pp. 852-865, Feb. 2018. doi:10.1109/TVT. 2017.2760367.

14. L. Y. Chen, H. Hopman, and R. R. Negenborn, "Distributed model predictive control for vessel train formations of cooperative multi-vessel systems," Transportation Research Part C-Emerging Technologies, vol. 92, pp. 101-118, May 2018. doi:10.1016/j.trc.2018. 04.013.

15. J. X. Zhang and G. H. Yang, "Fault-tolerant leader-follower formation control of marine surface vessels with unknown dynamics and actuator faults," Int. J. Robust Nonlinear Control, vol. 28, pp. 4188-4208, Apr. 2018. doi:10.1002/ rnc. 4228 .

16. M. Y. Fu and L. L. Yu, "Finite-time extended state observerbased distributed formation control for marine surface vehicles with input saturation and disturbances," Ocean Engineering, vol. 159, pp. 219-227, Apr. 2018. doi:10.1016/j. oceaneng.2018.04.016.

17. T. S. Li, R. Zhao, C. L. P. Chen, L. Y. Fang, and C. Liu, "Finite-time formation control of under-actuated ships using nonlinear sliding mode control," IEEE Transportation on Cybernetics, vol. 48, no. 11, pp. 3243-3253, Nov. 2018. doi:10.1109/TCYB.2018.2794968.

18. Z. J. Sun, G. Q. Zhang, Y. Lu, and W. D. Zhang, "Leaderfollower formation control of underactuated surface vehicles based on sliding mode control and parameter estimation," ISA Transactions, vol. 72, pp. 15-24, Nov. 2017. doi:10.1016/j.isatra.2017.11.008.

19. S. L. Dai, S. D. He, H. Lin, and C. Wang, "Platoon formation control with prescribed performance guarantees for USVs," IEEE Transportation on Industrial Electronics, vol. 65, no. 5, pp. 4237-4246, May 2018. doi:10.1109/TIE.2017.2758743.

20. Y. Lu, G. Q. Zhang, Z. J. Sun, and W. D. Zhang, "Robust adaptive formation control of underactuated autonomous surface vessels based on MLP and DOB," Nonlinear Dynamics, vol. 94, pp. 503-519, Jun. 2018. doi:10.1007/ s11071-018-4374-Z.

21. Y. Li and J. Zheng, "The design of ship formation based on a novel disturbance rejection control," International Journal of Control, Automation and Systems, vol. 16, no. 4, pp. 1833-1839, Feb. 2018. doi: 10.1007/s12555-017-0424-4.

22. B. S. Park and S. J. Yoo, "Adaptive-observe-based formation tracking of networked uncertain underactuated surface vessels with connectivity preservation and collision avoidance," Journal of the Franklin Institute-Engineering and Applied Mathematics, vol. 356, pp. 7947-7966, Apr. 2019. doi:10.1016/j.jfranklin.2019.04.017.

23. C. F. Huang, X. K. Zhang, and G. Q. Zhang, "Improved decentralized finite-time formation control of underactuated USVs via a novel disturbance observer," Ocean Engineering, vol. 174, pp. 117-124, Jan. 2019. doi:10.1016/j.oceaneng.2019.01.043.

24. Z. H. Peng, N. Gu, Y. Zhang, Y. J. Liu, D. Wang, and L. Liu, "Path-guided time-varying formation control with collision avoidance and connectivity preservation of under-actuated autonomous surface vehicles subject to unknown input gains," Ocean Engineering, vol. 191, pp. 1-10, Oct. 2019. doi:10.1016/j.oceaneng.2019.106501.

25. J. Li, J. L. Du, and W. J. Chang, "Robust time-varying formation control for underactuated autonomous underwater vehicles with disturbances under input saturation," Ocean Engineering, vol. 179, pp. 180-188, Mar. 2019. doi:10.1016/j.oceaneng.2019.03.017.

26. H. N. Esfahani, R. Szlapcznski and H. Ghaemi, "High performance super-twisting sliding mode control for a maritime autonomous surface ship (MASS) using ADPbased adaptive gains and time delay estimation", Ocean Engineering, vol. 191, no. 106526, pp.1-19, Nov. 2019. doi:10.1016/j.oceaneng.2019.106526.

27. T. Fossen, "Handbook of Marine Craft Hydrodynamics and Motion Control”, New York: Wiley, 2011.

28. E. Panteley, E, Lefeber, A. Loria and H. Nijmeijer, "Exponential tracking control of a mobile car using cascaded approach," IFAC Proceedings, vol. 31, no. 27, pp. 201-206, 1998. doi: 10.1016/S1474-6670(17)40028-0.

29. H. K. Khalil, "Nonlinear Systems" 3rd ed., New Jersey: Prentice Hall, 2002.

30. K. D. Do and J. Pan, "Global robust adaptive path following of underactuated ships," Automatica, vol. 42, no. 10, pp.1713-1722, Oct. 2006. doi: 10.1016/j. automatica.2006.01.026. 


\title{
CONTACT WITH THE AUTHORS
}

\author{
Tao Qin \\ e-mail:heu_qt@163.com
}

School of Mechanical Engineering

Hubei University of Arts and Sciences

Xiangyang, No.296

Longzhong Road, 441053,

China

\section{Zaopeng Dong}

e-mail:dongzaopeng@whut.edu.cn

Key Laboratory of High Performance Ship Technology

(Wuhan University of Technology)

Ministry of Education

Wuhan University of Technology

Wuhan, No. 1178

Heping Avenue 430063

\section{China}

School of Transportation

Wuhan University of Technology

Wuhan, No. 1178

Heping Avenue, 430063

China 